\title{
SARS-CoV-2 re-positivity within the first 3 months of COVID-19 recovery; probable re-infection
}

\author{
sara sadr ${ }^{1}$, Melika Arab Bafrani ${ }^{2}$, Alireza Abdollahi ${ }^{2}$, SeyedAhmad SeyedAlinaghi², \\ Esmaeil Mohammadnejad ${ }^{2}$, Roghieh Hossienzade ${ }^{2}$, Fereshteh Shahmari Golestan ${ }^{2}$, Zahra \\ Ahmadinejad $^{2}$, Mohammadreza Salehi ${ }^{3}$, Mohammad Javaherian ${ }^{2}$, Elahe kimyaee ${ }^{2}$, Fatemeh \\ Jafari $^{4}$, and Fereshteh Ghiasvand ${ }^{2}$ \\ ${ }^{1}$ Mazandaran University of Medical Sciences \\ ${ }^{2}$ Tehran University of Medical Sciences \\ ${ }^{3}$ Imam Khomeini Hospital Complex \\ ${ }^{4}$ Iran University of Medical Sciences
}

May 16, 2021

\begin{abstract}
Objectives Possibility of reinfection with SARS-CoV-2 changes our view on herd immunity and vaccination, and can impact worldwide quarantine policies. We performed RT-PCR follow-up studies on recovered patients to assess possible development of reinfections and re-positivity. Method During a 6-month period, 202 PCR-confirmed recovering COVID-19 patients entered this study. Follow-up RT-PCR tests and symptoms assessment were performed one month after the initial Positive results. patients who tested negative were tested again one and three months later. The Serum IgG and IgM levels were measured in the last follow-up session. Results In the first two follow-up sessions, 82 patients continued their participation, of which four patients tasted positive. In the second follow-up 44 patients participated, three of whom tested positive. None of the patients who tested positive in the first and second follow-up session were symptomatic. In the last session, 32 patients were tested and four patients were positive, three of them were mildly symptomatic and all of them were positive for IgG. Conclusion A positive RT-PCR in a recovering patient may represent reinfection. While we did not have the resources to prove reinfection by genetic sequencing of the infective viruses, we believe presence of mild symptoms in the three patients who tested positive over 100 days after becoming asymptomatic, can be diagnosed as reinfection. The IgG may have abated the symptoms of the reinfection, without providing complete protection.
\end{abstract}

\section{SARS-CoV-2 re-positivity within the first 3 months of COVID-19 recovery; probable re- infection}

\section{Running title:}

SARS-CoV-2 re-positivity, 3-months post-remission

\section{Author names and affiliations:}

Sara Sadr $^{1}$, Melika Arab Bafrani ${ }^{2}$, Alireza Abdollahi ${ }^{3}$, SeyedAhmad SeyedAlinaghi ${ }^{4}$, Esmaeil Mohammadnejad $^{5}$, Roghieh Hossienzade ${ }^{6}$, Fereshteh Shahmari Golestan ${ }^{7}$, Zahra Ahmadinejad ${ }^{8}$, Mohamadreza Salehi ${ }^{9}$, Mohammad Javaherian ${ }^{10}$, Elahe Kimyaee ${ }^{11}$, Fatemeh Jafari ${ }^{12}$, Fereshteh Ghiasvand ${ }^{8 *}$

1. School of medicine, Mazandaran University of Medical Sciences, Sari, Iran.

2. Students's scientific Research Center (SSRC), school of medicine, Tehran University of Medical Sciences, Tehran, Iran. 
3. Department of Pathology, school of medicine, Imam Khomeini Hospital Complex, Tehran University of Medical Sciences, Tehran, Iran.

4. Iranian Research Center for HIV/AIDS, Iranian Institute for Reduction of High Risk Behaviors, Tehran University of Medical Sciences, Tehran, Iran

5. Assistant Professor of Nursing Education, Department of Medical-Surgical Nursing and Basic Science, School of Nursing and Midwifery, Tehran University of Medical Sciences, Tehran, Iran.

6. MSc, Student in nursing management leader ship, Imam Khomeini Hospital Complex, Tehran University of Medical Sciences, Tehran, Iran.

7. Nurse Working in Department of Infectious Diseases, Tehran University of Medical Sciences, Tehran, Iran.

8. Liver Transplantation Research Center, Department of Infectious Diseases, Imam Khomeini Hospital Complex, Tehran University of Medical Sciences, Tehran, Iran.

9. Department of Infectious Diseases, Imam Khomeini Hospital Complex, Tehran University of Medical Sciences, Tehran, Iran.

10. Department of Physiotherapy, School of Rehabilitation, Liver Transplantation Research Center, Imam Khomeini Hospital Complex, Tehran University of Medical Sciences, Tehran, Iran.

11. Central Laboratory, Imam Khomeini Hospital Complex, Tehran University of Medical Sciences, Tehran, Iran.

12. School of medicine, Iran University of Medical Sciences, Tehran, Iran.

\section{*Corresponding author:}

Fereshteh Ghiasvand, M.D.

Liver Transplantation Research Center, Department of Infectious Diseases, Imam Khomeini Hospital Complex, Keshavarz boulevard, Tehran University of Medical Sciences, Tehran, Iran.

Tel: +982161192659 ,

Fax: +98 2166581657 ,

E-mail: ghiasvand_62@yahoo.com

\section{Authors' emails:}

Sara Sadr: sadrsara.md@gmail.com Melika Arab Bafrani: melika.arab92@gmail.com Alireza Abdollahi: alireza.a1345@yahoo.com SeyedAhmad SeyedAlinaghi: s.a.alinaghi@gmail.com Esmaeil Mohammadnejad: asreno1358@yahoo.com Roghieh Hossienzade: hroghieh@gmail.com Fereshteh Shahmari Golestan: fshahmari441@gmail.com Zahra Ahmadinejad: ahmadiz@tums.ac.ir Mohamadreza Salehi: salehi.mohamad3@gmail.com Mohammad Javaherian: javaherian_m@razi.tums.ac.ir Elahe kimyaee: e.kimyaee1991@gmail.com Fatemeh Jafari: fatemejafari72@gmail.com Fereshteh Ghiasvand: ghiasvand_$62 @$ yahoo.com

\section{Acknowledgements}

This research has been financially supported by Tehran University of Medical Sciences \& health services grant [grant number: 99-1-101-47279]. The supporting institution had no involvement in study design; in the collection, analysis and interpretation of data; in the writing of the report; and in the decision to submit the article for publication. Authors also would like to appreciate the support and constructive comments of the methodologist(s) research development office, Imam khomeini Hospital complex. We would like to thank our patients for their time and cooperation, and also the medical staff of the Imam Khomeini Hospital Complex who helped facilitated the process of our research.

\section{Conflict of Interest: none}

\section{Abstract:}

Objectives 
Possibility of reinfection with SARS-CoV-2 changes our view on herd immunity and vaccination, and can impact worldwide quarantine policies. We performed RT-PCR follow-up studies on recovered patients to assess possible development of reinfections and re-positivity.

Method

During a 6-month period, 202 PCR-confirmed recovering COVID-19 patients entered this study. Followup RT-PCR tests and symptoms assessment were performed one month after the initial Positive results. patients who tested negative were tested again one and three months later. The Serum IgG and IgM levels were measured in the last follow-up session.

Results

In the first two follow-up sessions, 82 patients continued their participation, of which four patients tasted positive. In the second follow-up 44 patients participated, three of whom tested positive. None of the patients who tested positive in the first and second follow-up session were symptomatic. In the last session, 32 patients were tested and four patients were positive, three of them were mildly symptomatic and all of them were positive for IgG.

Conclusion

A positive RT-PCR in a recovering patient may represent reinfection. While we did not have the resources to prove reinfection by genetic sequencing of the infective viruses, we believe presence of mild symptoms in the three patients who tested positive over 100 days after becoming asymptomatic, can be diagnosed as reinfection. The IgG may have abated the symptoms of the reinfection, without providing complete protection.

\section{Keywords:}

COVID-19, Reinfection, Virus Shedding, Polymerase Chain Reaction, Seroconversion

\section{Introduction}

Since November 2019, the Severe Acute Respiratory Syndrome Coronavirus 2 (SARS-CoV-2) has infected more than 134 million people worldwide and has caused more than 2 million deaths [1]. No curative drug or specific treatment is known to have considerable efficacy against this infection; but, currently over 200 candidate vaccines have been acknowledged by the WHO [2-4].

Our experience with other respiratory infections, such as those caused by influenza and seasonal respiratory infections caused by coronavirus lead us to believe that the natural immunity after an episode of infection with this virus may not cause long-time immunity [5]. Some studies have shown a substantial wane in antibody levels within a few months after remission [6,7]; and the studies that report re-infection further question the efficacy and longevity of the acquired immunity after infection with this virus. Several cases of suspected and proven instances of re-infection with SARS-CoV-2 have been reported in immunocompetent patients in different age groups, in patients with different levels of antibody response [8-10].

The duration of sustained antibody response after an episode of infection is generally a representative of the immune protection that can be achieved by vaccination against the same virus [11]. Waning of the IgG antibodies that are naturally produced in a patient after infection with SARS-CoV-2, undermines the supposed efficacy of vaccination; especially since cases of symptomatic re-infection with replication-competent virus have been reported within the first 6 months after the initial infection [12, 13].

To this date, thousands of distinct variants of SARS-CoV-2 have been identified with over 400 variants in the spike protein, which is presumably the binding cite of neutralizing antibodies [14]. Besides, in many cases of re-infection, genetic sequencing has revealed a different clade of the virus to be the causative pathogen [15-18]. We can assume - as is the case with infections caused by rhinoviruses and influenza - that the protective activity of antibodies is limited to each specific subtype of the virus, this can possibly explain why re-infection can occur in presence of detectable levels of $\operatorname{IgG}[19,20]$. 
In this study we investigated the rate of symptomatic and asymptomatic re-positivity with SARS-CoV-2 in recovering patients for up to four months after the initial diagnosis of Coronavirus Disease 2019 (COVID-19); and find possible risk factors that are associated with re-infection.

\section{Methods}

Study design and participants

This prospective study was performed for a duration of six months from May to September of 2020 on Coronavirus Disease 2019 (COVID-19) patients who were admitted to the Imam Khomeini Referral Hospital, Tehran, Iran.

\section{Eligibility Criteria}

Patients who had been diagnosed with COVID-19 (approved by a Real-Time Polymerase Chain Reaction (RT-PCR) test of nasopharyngeal specimens) and had been admitted based on the national criteria for hospitalization (a sustained peripheral oxygen saturation of under $93 \%$ and/or a respiratory rate of over $30 /$ minute or sustained nausea and vomiting and severe weakness even with normal oxygen saturation), were brought into the study upon discharge. The current national discharge criteria dictate that prior to discharge patients must have at least two consecutive afebrile days with a blood oxygen saturation of over $90 \%$, oral intake without nausea, and improvement in weakness. The exclusion criteria were a negative RT-PCR result or lack of documentation of a positive result at the initial hospitalization.

\section{Study initiation and follow-up sessions}

All information regarding patients' admission and epidemiological data including age, sex, any past medical history and resent use of immunocompromising medications were documented.

The first follow-up RT-PCR study was performed one month after the initial positive RT-PCR test; at which point all patients had been asymptomatic for at least 14 days. All patients who tested negative were re-tested one and three months after the date of the first follow-up RT-PCR test (Figure-1). A complete assessment of signs and symptoms related to COVID-19 along with serology testing for anti-SARS-CoV-2 IgG and IgM levels were also performed during the $3^{\text {rd }}$ follow-up visit.

\section{$R T-P C R$}

A nasopharyngeal swab sample was obtained by a trained technician and RNA was extracted with a Real Genomics viral nucleic acid extraction kit (Cat.No.YVN50/YVN100). The Novel Coronavirus (2019-nCOV) Nucleic Acid Diagnostic Kit (PCR-Fluorescence Probing), Sansure Biotech (S3102E), made in Changsha, China was used for qualitative detection of the ORF1ab and N genes of 2019-nCOV with a cycle threshold of less than $35(\mathrm{Ct}<35)$ for positive control and $\mathrm{Ct}>40$ for negative control at channel FAM, ROX and CY5 (internal control) according to kit instructions.

\section{Antibodies}

A 5cc whole blood sample was drawn (without anticoagulants), and serum was derived from the specimen using centrifugation (3000xg for 10 minutes). The Enzyme-linked Immunosorbent Assay (ELISA) method (Pishtaz Teb SARS-CoV-2 IgM and IgG- Iran) was used to test the serum antibody levels. The test was performed according to the manufacturer's brochure. Results greater than 1.1 were considered positive and those less than 0.9 as negative. Results within the mentioned range were reported as borderline and the test was redone on a second, fresh serum sample to confirm the initial results.

The manufacture reported diagnostic specifications of the test kits:

Specificity: $97.30 \%$ and Sensitivity: $79.40 \%$ for SARS-COV-2-IGM kit.

Specificity: $98.30 \% \%$ and sensitivity: $91.10 \%$ for SARS-COV-2-IGG kit.

Measurements and statistical analysis 
Data was analyzed using SPSS software V.22.0. Quantitative variables are reported by mean and standard deviation (SD) and qualitative variables are reported using frequency and percentage. Chi-square and Fisher's exact tests were used to assess the statistical relationships between categorical variables. The level of significance was set as $\mathrm{P}$-value $<0.05$ for all analyses.

Ethical considerations

Informed consent was obtained from participants and they were advised that they can leave the study at any time point and this will not hinder their current treatment or future visits to the hospital and the quality of care they would receive. This study was conducted in accordance with the 1964 Helsinki Declaration and approved by the Tehran University of Medical Sciences ethics board committee (Ethics code: IR.TUMS.VCR.REC.1399.076).

\section{Results}

\section{One month after the first positive RT-PCR (1st F/U)}

Overall, 202 discharged patients were contacted for follow-up laboratory studies and 82 patients, including 27 women $(33 \%)$ and 55 men $(64 \%)$, decided to participate in this study.

Patients had a median age of 47 ranging between 29 and 84 years old. Table 1 shows the demographic description of participants. The first RT-PCR results of 4 patients $(4.87 \%)$ were positive; who were asymptomatic and not different from those who tested negative in terms of the duration of the initial admission, the severity of the first episode of the disease, presence and type of underlying diseases, and recent history of using immunosuppressive drugs (P-value: $0.63,0.57,0.59,0.61$ ).

\section{Two months after the first positive RT-PCR (2nd F/U)}

One month after the first negative follow-up result, among the 78 patients who had tested negative, 44 patients $(53.65 \%)$ continued their participation in the study. Additional data regarding patients who tested positive are presented in Figure-1. The patients who tested positive were not different in terms of the duration of the initial admission, severity of the disease, and the need for ICU admission (P-value: 0.69, 0.62 , and 0.65$)$.

\section{Four months after the first positive RT-PCR (3rd F/U)}

A mong the 41 patients who had tested negative, 32 patients $(39.02 \%)$ continued their participation in the study. Four patients had a positive RT-PCR result (12.5\%). None of the patients who tested positive were symptomatic in previous studies; but now three of them were mildly symptomatic; also, two patients had underlying medical conditions (Figure-1). None of the patients who tested negative were symptomatic. The underlying medical conditions, history of using immunosuppressive drugs, initial disease severity, admission duration and history of ICU admission in the first episode of infection were not significantly different between patients who had a positive RT-PCR result and those who did not (P-value: 0.67, 0.59, 0.64, 0.64, 0.71).

The IgM levels were $<1.1 \mathrm{~g} / \mathrm{L}$ in 30 (94\%) patients (negative); and positive in 2 patients. The patients who tested positive were asymptomatic and did not have a positive RT-PCR test in any of the previous follow-up studies. The IgG levels were $>1.1 \mathrm{~g} / \mathrm{L}$ in 30 (94\%) patients (positive); and negative in 2 patients. The mean IgG level was $7.94 \pm 3.67 \mathrm{~g} / \mathrm{L}$ and ranged between 1.48-14.5 g/L. The two patients, who had a negative $\operatorname{IgG}$ test result, did not test positive in any of the follow-up RT-PCR tests.

All patients recovered with no further complications and none of the patients who re-tested positive required readmission or further medical treatment.

\section{Discussion}

\section{Interpretations of a positive RT-PCR re-test}

Considering the high infection rate, few cases of reinfection with SARS-CoV-2 have been reported. Often, for the diagnosis COVID-19 in a recovered individual a positive RT-PCR test with or without accompanying 
symptoms has been used. Although, in these cases different speculations for interpretation of a re-positivity can be made; each of which will have specific reverberations [21].

In some studies, a positive RT-PCR in a recovering patient who tested negative upon resolution of their symptoms, has been considered a strong indicator of reinfection [10]. Although, we should consider the possibility of a false result in either test [22]. A false positive result can occur in a recovering patient; also a false negative result shortly after subsidence of symptoms, followed by a correctly positive test misleads physicians towards a re-infection diagnosis [23]. To avoid this problem - as per WHO recommendations - in many regions the treatment protocol for COVID-19 requires two consecutive negative RT-PCR results prior to discharge [24, 25].

Also, RT-PCR cannot distinguish between replication-competent viruses and remnants of viral fragments that are expelled from a recovering patient $[26,27]$. Viral shedding from the respiratory tract during the recovery period has been reported to last for as long as 12 weeks after infection [28]; thus, a significant time-gap between the first episode of infection and a positive RT-PCR test can clarify that the patient has passed the viral shedding stage.

The first follow-up RT-PCR

As per national protocol, RT-PCR testing was not performed upon discharge to confirm viral clearance. The first follow-up RT-PCR study was performed 30 days after the first positive RT-PCR result in each patient, which was after at least 14 days of being asymptomatic. Considering the short interval between the onset of the disease and the test, no recorded negative RT-PCR result at discharge, and the fact that the patients who tested positive were asymptomatic, we believe that the positive results show continued viral shedding rather than re-infection. The four patients who tested positive were not significantly different from others in terms of the severity of the initial illness, duration of the first admission, age, or underlying conditions to suggest a risk factor for a longer viral shedding period. Other studies have reported that up to $14 \%$ of recovering asymptomatic patients who tested negative upon discharge, re-test positive [29]. Other studies have reported patients who became symptomatic after a short symptom-free period. A 24-year-old healthworker became symptomatic and tested positive for SARS-CoV-2 within 52 days of an initial symptomatic diagnosis of COVID-19. Similar to our study, no confirmatory RT-PCR testing was performed at discharge. Serum antibodies were not detected at the beginning of the second symptomatic period, which can represent an incomplete immune response that left the infection temporarily dormant, only to be re-activated again; and although the patient was symptomatic and had occupational contact with infected patients during her symptom-free period, re-activation was considered more probable than re-infection [30]. Similar studies have reported a return of mild or even severe symptoms; but within a short time frame and without genetic analysis of the infective pathogens, they were reported to be cases of reactivation rather than re-infection [31-34].

The second follow-up RT-PCR

Among the 44 patients who tested negative in the first test and continued their cooperation with the study, 3 patients (6.81\%) tested positive 60 days after the initial infection (one month after the first negative RT-PCR test). These patients again were asymptomatic and the interval between the initial infection and this positive test is not significant enough to rule out viral shedding. Although, in case of viral shedding and/or presence of remnant viral particles, we would have expected to achieve a positive result in the previous test as well as this one; but this dissonance can be explained in absence of re-infection. Given the limited accuracy of RT-PCR, in case of a false negative in the first follow-up test or a false positive in the second test, continued shedding or complete recovery (respectively) can be misdiagnosed as re-infection [35, 36]. On the other hand, studies have shown that COVID-19 patients have a lower concentration of ACE2 monocyte expression-the endogenous entry receptor of SARS-CoV-2 - and researchers hypothesize that the virus can remain dormant in peripheral blood mononuclear cells and cause a relapse after the respiratory system has been cleared of the virus and patient has tested negative [37-39].

Positive RT-PCR tests in absence of significant symptoms in a recovering patient has also been reported in 
many studies [18, 40]. In some cases, symptoms were present, although less severe than the first episode [41]. Our findings include asymptomatic and mildly symptomatic patients who re-tested positive for SARS-CoV-2. None of our cases had symptoms as or more severe than the first episode of infection. Contrasting our results, the majority of other reports have described more severe symptoms in patients who re-tested positive [15-17, 42] and researchers have hypothesized that a selection bias towards testing and confirming re-infection in symptomatic patients [43] and/or a primed/heightened immune response upon the second episode of infection can be the reason why most cases of re-infection pertain to patients with intense symptoms upon second exposure and infection $[15,42]$.

An episode of re-infection can be proved by a positive viral culture [21, 44] and/or genetic sequencing of the infective virus in both episodes of infection to confirm that the second episode is caused by a different clade of the virus. Tillett et al, reported a case of re-infection in a 25-year-old male, who recovered from a RT-PCR-confirmed episode of COVID-19, only to become symptomatic again after a 30-day symptomfree period. Genetic sequencing showed distinct genetic differences between the two specimen, indicating two separate instances of infection with genetically different variants of the virus [15]. Similar cases of re-infection with a genetically different clade of the virus within 6 months of an original episode of COVID-19 have been reported [16-18].

Unfortunately, genetic sequencing is not readily available and based on CDC recommendations in absence of genetic proof of infection with a different clade of the virus, a positive RT-PCR test that has been obtained after the first 90 days of the onset of the initial infection can be considered indicative of re-infection. Although, a positive RT-PCR test after two consecutive negative results, especially if accompanied by typical symptoms, can be defined as re-infection even within the first 90 days of the first episode of infection [21].

The third follow-up RT-PCR

According to the recommended CDC definitions [28], re-infection is a probable diagnosis for the three symptomatic patients who tested positive in the last RT-PCR test; but it is less likely in case of the one asymptomatic patient. The third RT-PCR screening was performed 120 days after the diagnosis of COVID-19 in 32 patients (who had tested negative on both of the previous tests). Although, the symptoms could have been caused by re-activation of dormant infection and release of viruses from body reservoirs, they could also be caused by re-infection. A similar process involving latent infection of cells followed by transcription of viral genome has also been suggested, which would result in reactivation of the virus from a latent to a lytic stage after a symptom-free period, causing a resurgence of COVID-19 symptoms [39]; but the long interval between the two positive RT-PCR results makes re-activation an unlikely diagnosis [21]. These four patients were not significantly different from those who tested negative in terms of past medical history or severity of the initial episode of COVID-19. Also, we did not find any risk factors that could help distinguish patients who are more susceptible to re-infection from those who are not.

\section{Humoral response}

Both insufficient and overactive immune responses have been reported in COVID-19 patients [45]. Production of protective cytokines and IFN-gamma, which is mediated by the CD4+ and CD8+ cells, plays an important role in containing and resolution of the infection [46]. The dynamics of the antibody response in COVID-19 patients is not completely known; and different studies have reported different rates of seroconversion. Zhao et al [47] and Liu et al [48] reported seroconversion in all infected patients respectively by 39 and 14 days after the onset of infection. Liu et al also reported that by the $60^{\text {th }}$ day IgM antibodies were undetectable in about one-third of the patients and the IgG titers had decreased substantially [48]. Based on another study, although recently discharged patients have a high level of humoral immunity against the virus, antibodies start to decreases within 2 to 3 months after the infection [13]. In another study the seroconversion rate for $\operatorname{IgG}$, $\operatorname{IgM}$ and $\operatorname{IgA}$ was $\sim 90 \%$ and most patients seroreverted within 75 days; with IgG levels remaining detectable over 90 days after the symptom onset in more than $99 \%$ of patients [49]. Multiple studies have also concluded that the humoral immunity against this virus could be short-lived [50]. Contrasting these studies, our results showed that $94 \%$ of patients were positive for neutralizing antibodies (IgG) 120 days 
after the onset of symptoms; which is in line with the results of an Icelandic population study that reported a 91\% seropositivity four months after the initial diagnosis of COVID-19 [6]. In evaluation of the results of these studies, we should take into account the natural process of the humoral response. In case of many other viral infections - where seroconversion is sustained as seromaintenance and immunity - we see a temporary decrease of antibody levels during the first few months of infection/inoculation [51], and since the emergence of COVID-19 is recent, we cannot judge the humoral response to this virus in long term and a rebound increase in antibody levels can be expected [50].

In our study, the four patients who had a positive result in RT-PCR screening 120 days after the initial diagnosis of COVID-19, were also positive for neutralizing antibodies; and although they theoretically may have prevented a severe episode of re-infection and caused a lack of any symptoms in one RT-PCR-positive patient, we cannot know for sure if those levels are high enough to be completely protective [21]. In a similar study Zhang et.al reported re-infection in 6 recovered patients that was caused by viruses from lineages different from the first infection. All these patients had varied levels of antibodies and they concluded that presence and even maintenance of the humoral response cannot rule out the possibility of re-infection [52]. We also believe that the two patients who did not have sufficient levels of $\operatorname{IgG}(<1.1 \mathrm{~g} / \mathrm{L})$, have been protected from an episode of re-infection by a strong cellular immune response, even within an epidemic situation.

Genetic sequencing can rule out a false positive in RT-PCR testing and determine if the infection is caused by a different subclass of the virus. In cases of re-infection with a different clade of the virus, even protective levels of IgG may not be effective [21]. We hypothesize that high levels of neutralizing antibodies do not make the diagnosis of re-infection unlikely, unless there is genetic proof that the positive RT-PCR results are related to the same strain of the virus from the first episode; in which case re-activation/relapse would be a more likely diagnosis.

\section{Limitations}

Some of the limitations of our study were that a considerable number of participants dropped out during the study, this study was only performed in one medical center, and that we could not perform genetic sequencing to prove infection with a different clade of the virus, or cultures to prove presence of replication-competent virus. The diagnosis of re-infection in our study is based on RT-PCR results, typical symptoms and the long interval between the two positive results. And, since we did not measure the viral load in patients who tested positive, the diagnosis of re-infection in our study is as certain as is the specificity of the RT-PCR test. We were also unable to perform required serology testing from the beginning of the study.

\section{Conclusion}

Based on current diagnostic guidelines, re-infection associated with mild symptoms was detected in 3 out of 82 patients; barring possible false positive results. We cannot confirm re-infection without positive viral cultures. We also believe that the quantity of antibodies that are produced against this virus can be sustained longer than the initial studies suggest, although the protective abilities of these antibodies against infection with the same or a different subtype of the virus needs to be studied further. The emergence of new vaccines against this virus, is a considerable achievement with limited guaranteed outcomes, because the intricacies of the long-term immune response against this virus is not fully known, and further studies on cases of supposed re-infection are needed to clarify the probability and underlying risk factors of re-infection.

\section{Declarations of interest: none}

\section{Refrences}

1. Organization, W.H. WHO Coronavirus Disease (COVID-19) Dashboard . 2021 February 4th, 2021; Available from: https://covid19.who.int/.

2. Shang, W., et al., The outbreak of SARS-CoV-2 pneumonia calls for viral vaccines. npj Vaccines, 2020. 5 (1): p. $1-3$. 
3. Ayotte, K., J. Gerberding, and J.S. Morrison, Ending the Cycle of Crisis and Complacency in US Global Health Security: A Report of the CSIS Commission on Strengthening America's Health Security . 2020: Center for Strategic \& International Studies.

4. WHO, Draft landscape of COVID-19 candidate vaccines. World Health Organisation, 2020.

5. Cohen, J.I. and P.D. Burbelo, Reinfection with SARS-CoV-2: Implications for Vaccines. Clinical Infectious Diseases, 2020.

6. Gudbjartsson, D.F., et al., Humoral immune response to SARS-CoV-2 in Iceland. New England Journal of Medicine, 2020.383 (18): p. 1724-1734.

7. Crawford, K.H., et al., Dynamics of neutralizing antibody titers in the months after SARS-CoV-2 infection. MedRxiv, 2020.

8. Dickson, M., R. Mathews, and G.G. Menon, ANALYSIS OF COVID-19 REINFECTION RATES AND ITS UNDERLYING CAUSES: A SYSTEMATIC REVIEW. 2020.

9. Krishna, E., et al., COVID-19 reinfection: Linked possibilities and future outlook. Journal of Family Medicine and Primary Care, 2020.9 (11): p. 5445.

10. Osman, A., M. Al Daajani, and A. Alsahafi, Re-positive coronavirus disease 2019 PCR test: could it be a reinfection? New Microbes New Infect . 2020.

11. Plotkin, S.A., Correlates of protection induced by vaccination. Clinical and vaccine immunology, 2010. 17 (7): p. 1055-1065.

12. Seow, J., et al., Longitudinal evaluation and decline of antibody responses in SARS-CoV-2 infection. MedRxiv, 2020.

13. Long, Q.-X., et al., Clinical and immunological assessment of asymptomatic SARS-CoV-2 infections. Nature medicine, 2020.26 (8): p. 1200-1204.

14. Koyama, T., D. Platt, and L. Parida, Variant analysis of SARS-CoV-2 genomes. Bulletin of the World Health Organization, 2020.98 (7): p. 495.

15. Tillett, R.L., et al., Genomic evidence for reinfection with SARS-CoV-2: a case study. The Lancet Infectious Diseases, 2021.21 (1): p. 52-58.

16. Prado-Vivar, B., et al., COVID-19 Re-Infection by a Phylogenetically Distinct SARS-CoV-2 Variant, First Confirmed Event in South America. First Confirmed Event in South America.(September 3, 2020), 2020.

17. Van Elslande, J., et al., Symptomatic SARS-CoV-2 reinfection by a phylogenetically distinct strain. Clin Infect Dis, 2020.10 .

18. Scaria, V., Asymptomatic reinfection in two healthcare workers from India with genetically distinct SARSCoV-2. 2020.

19. Mo, H., et al., Longitudinal profile of antibodies against SARS-coronavirus in SARS patients and their clinical significance. Respirology, 2006. 11 (1): p. 49-53.

20. Payne, D.C., et al., Persistence of antibodies against Middle East respiratory syndrome coronavirus. Emerging infectious diseases, 2016. 22 (10): p. 1824.

21. Yahav, D., et al., Definitions for coronavirus disease 2019 reinfection, relapse and PCR re-positivity. Clin Microbiol Infect, 2020.

22. Arevalo-Rodriguez, I., et al., False-negative results of initial RT-PCR assays for COVID-19: a systematic review. PloS one, 2020. 15 (12): p. e0242958. 
23. Luo, A., Positive SARS-Cov-2 test in a woman with COVID-19 at 22 days after hospital discharge: A case report. Journal of Traditional Chinese Medical Sciences, 2020. 7 (4): p. 413-417.

24. Organization, W.H., Criteria for releasing COVID-19 patients from isolation: scientific brief, 17 June 2020 . 2020, World Health Organization.

25. Tao, W., et al., Re-detectable positive SARS-CoV-2 RNA tests in patients who recovered from COVID-19 with intestinal infection.Protein \& cell, 2020: p. 1-6.

26. Kang, H., et al., Retest positive for SARS-CoV-2 RNA of "recovered" patients with COVID-19: Persistence, sampling issues, or re-infection? Journal of medical virology, 2020. 92 (11): p. 2263-2265.

27. Chen, Y., et al., Re-evaluation of retested nucleic acid-positive cases in recovered COVID-19 patients: Report from a designated transfer hospital in Chongqing, China. Journal of infection and public health, 2020. 13 (7): p. 932-934.

28. Control, C.f.D. and Prevention, Duration of isolation and precautions for adults with COVID-19. 2020 . 2020 .

29. Lu, J., et al., Clinical, immunological and virological characterization of COVID-19 patients that test re-positive for SARS-CoV-2 by RT-PCR. EBioMedicine, 2020. 59 : p. 102960.

30. Bonifacio, L.P., et al., Are SARS-CoV-2 reinfection and Covid-19 recurrence possible? a case report from Brazil. Revista da Sociedade Brasileira de Medicina Tropical, 2020. 53 .

31. Ye, G., et al., Clinical characteristics of severe acute respiratory syndrome coronavirus 2 reactivation. Journal of Infection, 2020. 80 (5): p. e14-e17.

32. Ravioli, S., H. Ochsner, and G. Lindner, Reactivation of COVID-19 pneumonia: a report of two cases. The Journal of infection, 2020.

33. Kang, Y.J. and H. Joo, South Korea's COVID-19 infection status: From the perspective of reconfirmation after complete recovery. Journal of Pure and Applied Microbiology, 2020. 14 .

34. Loconsole, D., et al., Recurrence of COVID-19 after recovery: a case report from Italy. Infection, 2020: p. $1-3$.

35. Anwar, H. and Q.U. Khan, Pathology and Therapeutics of COVID-19: A Review. International Journal of Medical Students, 2020.8 (2): p. 113-120.

36. Xie, C., et al., False negative rate of COVID-19 is eliminated by using nasal swab test. Travel medicine and infectious disease, 2020.

37. Zhang, D., et al., COVID-19 infection induces readily detectable morphologic and inflammation-related phenotypic changes in peripheral blood monocytes. Journal of leukocyte biology, 2020.

38. Elberry, M.H. and H. Ahmed, Occult SARS-CoV-2 infection; a possible hypothesis for viral relapse. Medical hypotheses, 2020.144 : p. 109980.

39. Mondal, R., et al., Recurrence of COVID-19: Treading the Fine Line Between Relapse and Re-infection. International Journal of Medical Students, 2020. 8 (3): p. 311-313.

40. To, K.K.-W., et al., COVID-19 re-infection by a phylogenetically distinct SARS-coronavirus-2 strain confirmed by whole genome sequencing. Clinical infectious diseases, 2020.

41. Chen, X., et al., Disease severity dictates SARS-CoV-2-specific neutralizing antibody responses in COVID-19. Signal transduction and targeted therapy, 2020. 5 (1): p. 1-6.

42. Larson, D., et al., A Case of Early Re-infection with SARS-CoV-2. Clinical infectious diseases: an official publication of the Infectious Diseases Society of America, 2020. 
43. Iwasaki, A., What reinfections mean for COVID-19. The Lancet Infectious Diseases, 2021. 21 (1): p. $3-5$.

44. Gousseff, M., et al., Clinical recurrences of COVID-19 symptoms after recovery: viral relapse, reinfection or inflammatory rebound? Journal of Infection, 2020. 81 (5): p. 816-846.

45. Chen, Z. and E.J. Wherry, T cell responses in patients with COVID-19. Nature Reviews Immunology, 2020. 20 (9): p. 529-536.

46. Channappanavar, R., et al., Virus-specific memory CD8 T cells provide substantial protection from lethal severe acute respiratory syndrome coronavirus infection. Journal of virology, 2014.88 (19): p. 11034-11044.

47. Zhao, J., et al., Antibody responses to SARS-CoV-2 in patients with novel coronavirus disease 2019. Clinical infectious diseases, 2020. 71 (16): p. 2027-2034.

48. Liu, A., et al., Antibody responses against SARS-CoV-2 in COVID-19 patients. Journal of medical virology, 2021. 93 (1): p. 144-148.

49. Iyer, A.S., et al., Persistence and decay of human antibody responses to the receptor binding domain of SARS-CoV-2 spike protein in COVID-19 patients. Science immunology, 2020. 5 (52).

50. Baumgarth, N., et al., Antibody Responses to SARS-CoV-2: Let's Stick to Known Knowns. The Journal of Immunology, 2020.205 (9): p. 2342-2350.

51. Amanna, I.J., N.E. Carlson, and M.K. Slifka, Duration of humoral immunity to common viral and vaccine antigens. New England Journal of Medicine, 2007. 357 (19): p. 1903-1915.

52. Zhang, J., et al., COVID-19 reinfection in the presence of neutralizing antibodies. National Science Review, 2021.

\section{Author contributions statement:}

Sara Sadr: Writing - Original Draft, Melika Arab Bafrani: Investigation, Alireza Abdollahi: Conceptualization, Investigation, SeyedAhmad SeyedAlinaghi: Formal analysis, Esmaeil, Mohammadnejad: Data Curation, Roghieh Hossienzade: Investigation, Fereshteh Shahmari Golestan : Data Curation, Zahra Ahmadinejad: Writing - Review \& Editing, Mohamadreza Salehi: Supervision, Mohammad Javaherian: Investigation, Elahe kimyaee: Methodology, Fatemeh Jafari: Data Curation, Fereshteh Ghiasvand: Project administration and execution,

Table 1- Demographic data of participants

\section{Number of patients \\ Female \\ Male \\ Age \\ Underlying disease ${ }^{+}$:}

+ No patients in our study had a history of ESRD (End-Stage Renal Disease), chronic liver disease, neurological disorders, 


\section{Hosted file}

Figure.pdf available at https://authorea.com/users/414059/articles/522201-sars-cov-2-repositivity-within-the-first-3-months-of-covid-19-recovery-probable-re-infection

Hosted file

Table.pdf available at https://authorea.com/users/414059/articles/522201-sars-cov-2-repositivity-within-the-first-3-months-of-covid-19-recovery-probable-re-infection 